\title{
Potred Plouiaou(1490) et la question des chants de révolte en langue bretonne
}

Potred Plouiaou (1490) and the question of revolt songs in Breton

Donatien Laurent et Michel Nassiet

\section{(2) OpenEdition}

\section{Journals}

Édition électronique

URL : http://journals.openedition.org/abpo/3284

DOI : $10.4000 /$ abpo.3284

ISBN : 978-2-7535-5185-5

ISSN : 2108-6443

Éditeur

Presses universitaires de Rennes

Édition imprimée

Date de publication : 28 juillet 2016

Pagination : 27-54

ISBN : 978-2-7535-5183-1

ISSN : 0399-0826

\section{Référence électronique}

Donatien Laurent et Michel Nassiet, «Potred Plouiaou(1490) et la question des chants de révolte en

langue bretonne », Annales de Bretagne et des Pays de l'Ouest [En ligne], 123-2 | 2016, mis en ligne le 28 juillet 2018, consulté le 02 mai 2019. URL : http://journals.openedition.org/abpo/3284 ; DOI : 10.4000/ abpo.3284 


\title{
Potred Plouiaou (1490) et la question des chants de révolte en langue bretonne
}

\author{
Donatien LAURENT \\ Ethnologue, Directeur de recherches honoraire au CNRS, CRBC \\ Michel NASSIET \\ Professeur d'Histoire moderne, Université d'Angers, CERHIO UMR 6258, \\ Membre honoraire de l'Institut universitaire de France
}

Le présent travail ${ }^{1}$ est d'abord l'analyse critique et l'édition d'un texte qui nous paraît d'un intérêt historique exceptionnel, Potred Plouiaou (Les Gars de Plouyé), que La Villemarqué a publié en $1845^{2}$. Rappelons où en est la question du Barzaz-Breiz, qui commande les grands problèmes sur la littérature poétique orale en langue bretonne. Hersart de La Villemarqué, qui a publié en 1839 le premier grand corpus de chants bretons, en a établi les textes d'une manière qui ne satisfait pas la méthode scientifique, mais il a effectué un travail pionnier de collecte qui est remarquable à la fois en termes de quantité et de qualité. Sur les trois carnets de collecte qu'il a laissés, le premier a fait l'objet d'une édition critique ${ }^{3}$ et c'est celle-ci qui a montré la réalité et l'authenticité de cette collecte en milieu paysan illettré. La Villemarqué, en particulier, a noté plusieurs textes qui jamais plus n'ont été trouvés dans la tradition orale. Il reste à faire un travail analogue sur les deux derniers carnets en examinant de façon critique la provenance et la qualité de chacun des textes. C'est ce travail que nous faisons ici sur Potred Plouiaou, dont le manuscrit se trouve dans son deuxième carnet. Nous nous proposons de montrer qu'il s'agit bien d'une authentique collecte, et que ce

1. Ce travail a fait l'objet d'une communication au colloque international Cultures orales, histoires et mémoires des révoltes et contestations populaires ( $X V^{e}$-XVIII ${ }^{e}$ siècles), organisé par le CRHQ à l'université de Caen le 4 avril 2013. Nous remercions Eva Guillorel pour les relectures qu'elle a faites du présent texte et pour ses remarques stimulantes.

2. Hersart de La Villemarqué, Théodore, Barzaz-Breiz, Paris, Delloye, 1845, t. 2, p. 19.

3. LAURENT, Donatien, Aux sources du Barzaz-Breiz. La mémoire d'un peuple, Douarnenez, Ar Men, 1989. Pour une vue d'ensemble récente de la littérature orale bretonne, GuILLOREL, Eva, La complainte et la plainte. Chanson, justice, cultures en Bretagne (XVI $-X V I I I^{e}$ siècles), Rennes, PUR, 2010. NASSIET, Michel, "La littérature orale bretonne et l'histoire ", Annales de Bretagne et des Pays de l'Ouest, t. 106, 1999, nº 3, p. 35-64. 
chant rapporte un épisode de la révolte intervenue en 1490 en Cornouaille. C'est donc une démarche pluridisciplinaire qui est menée ici, combinant anthropologie, linguistique et histoire.

Les sources historiques sur cette révolte sont très limitées. La Villemarqué et Boris Porchnev n'en connaissaient qu'une seule, une page composée un siècle après l'événement par un chanoine de Quimper, Jean Moreau $^{4}$ († 1617), qui, écrivant pendant la guerre de la Ligue (1589-1598), était épouvanté par ce qu'il appelait la " paysantaille " de son temps. C'est sur cette source très laconique et partiale, hostile aux paysans, que Porchnev ${ }^{5}$ fonde son affirmation que ceux-ci auraient eu le projet d'anéantir la classe seigneuriale, un projet dont on ne trouve aucune trace d'un début de réalisation ${ }^{6}$.

Il est intéressant de rapprocher Les Gars de Plouyé de l'autre chant recueilli par La Villemarqué et qui provient du même contexte historique, Le Faucon, et même de le comparer à un chant en langue française composé en 1525 en Lorraine pendant la Guerre des Paysans. Ces trois textes présentent des traits communs et permettent de préciser la notion de chant de révolte.

Ces chants permettent enfin de contribuer à l'exploration du phénomène de mémoire collective des révoltes. Un enjeu historique de cette discussion est de montrer que la littérature orale en langue bretonne a pu transmettre une mémoire collective et même des textes précis à propos d'épisodes de révoltes sur des durées allant jusqu'à trois siècles et demi. Un autre enjeu, méthodologique celui-là, est de revenir sur le problème des chants qui n'ont été collectés que par un seul chercheur, puisque c'était, pour Luzel, un fort indice que ce fussent des faux.

\section{La Villemarqué et la poésie orale bretonne : bilan critique}

Sans raconter une fois de plus la querelle du Barzaz-Breiz, il nous paraît utile, dans un but de clarification, de distinguer ce qui, dans le travail de La

4. Moreau, Chanoine Jean, Histoire de ce qui s'est passé en Bretagne durant les guerres de la Ligue, Saint-Brieuc, Prud'homme, 1857, p. 16-21.

5. PORCHNEV, Boris, "Les buts et les revendications des paysans bretons lors de la révolte bretonne de 1675 ", publié dans ESB, Les Bonnets rouges, Paris, 10-18, 1975, p. 242244. Sur 1490, cf. p. 239 et p. 258. Pour une synthèse récente, AuBERT, Gauthier, Révoltes et répressions dans la France moderne, Paris, Colin, 2015.

6. Ainsi les déclarations d'héritages avoués au duc en Cornouaille ne révèlent-elles aucun décès de nobles à cette date (NASSIET, Michel, "Émeutes et révoltes en Bretagne pendant la guerre d'Indépendance (1487-1490) ", Actes du 114 Congrès national des Sociétés savantes (Paris, 1989), Section d'histoire médiévale et de philologie, Paris, CTHS, 1990, p. 137-156, notamment p. 152). Une critique récente du texte de Moreau va dans le même sens à propos de la mobilisation armée des paysans pendant la guerre de la Ligue (HAMON, Philippe, " La défaite ou le chaos. Les paysans bas-bretons à la bataille pendant les guerres de la Ligue sous le regard du chanoine Moreau ", dans BolTANSKI, Ariane, LAGAdEc, Yann, Mercier, Franck [dir.], La Bataille. Du fait d'armes au combat idéologique, $X I^{e}$-XIX siècle, Rennes, PUR, 2015, p. 143-155). 


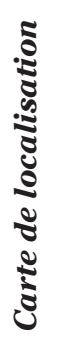

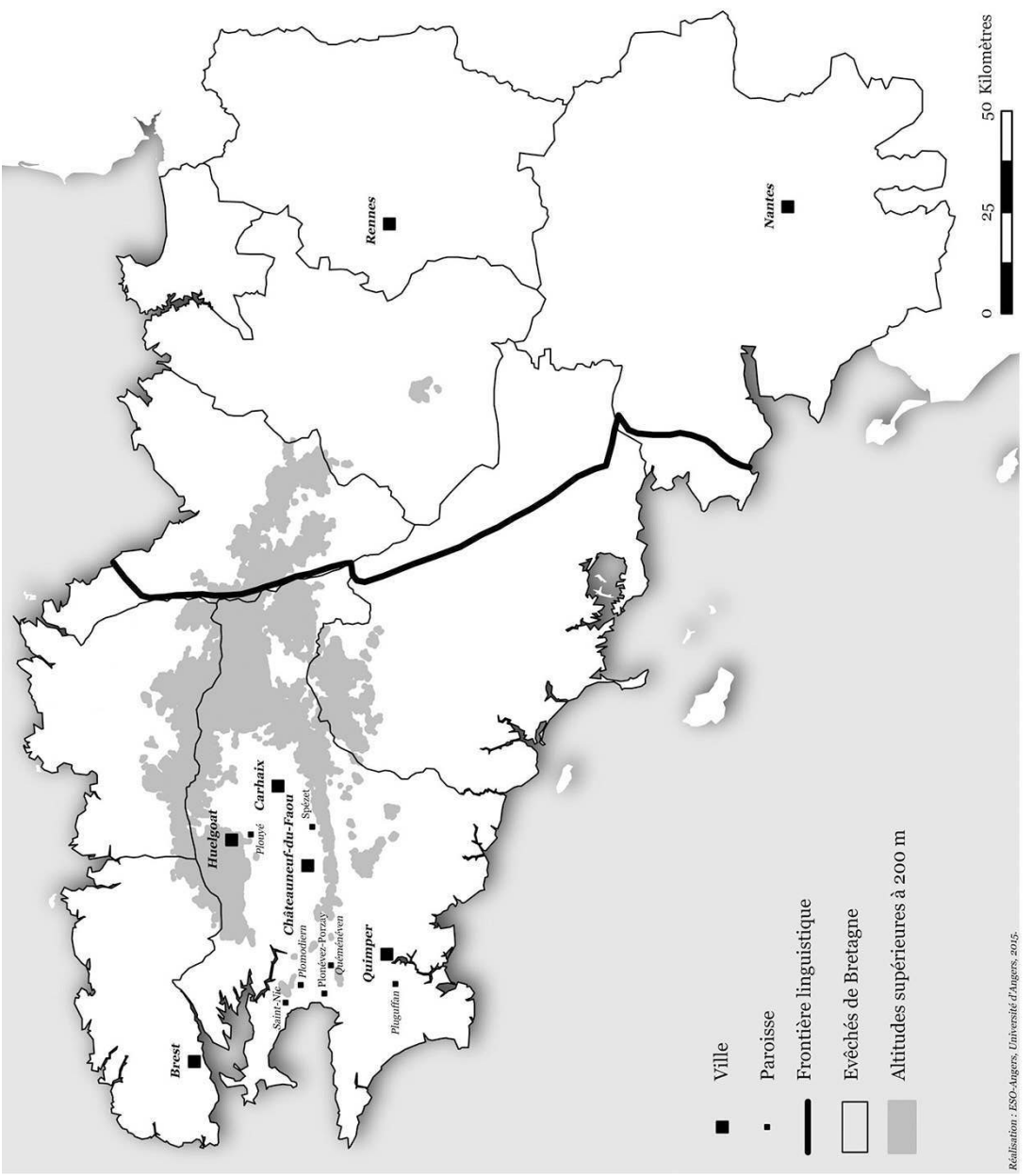


Villemarqué sur la poésie orale chantée, garde un caractère scientifique et ce qui en constitue une rupture.

Dans le premier carnet manuscrit, qui contient notamment le texte du Faucon, l'extrême rapidité de l'écriture, la fréquence des abréviations, la présence de fautes qui ne peuvent être imputables qu'à un chanteur, certains désordres dans les couplets, d'autres indices encore montrent tout d'abord qu'il s'agit de notations de terrain recueillies de la bouche d'un chanteur $^{7}$. Ces transcriptions montrent aussi que si le collecteur ignorait encore en effet l'orthographe usuelle du breton, encore mal définie, il comprenait fort bien ${ }^{8}$ la langue vernaculaire de son pays de naissance. Connaître une langue, dit Saussure ${ }^{9}$, c'est la comprendre et la parler, sans que se pose la question de son écriture, car la langue est un objet oral, absolument distinct de l'écriture - même si on les confond trop souvent. Ce premier carnet montre que le jeune La Villemarqué a eu une intense activité de collecteur dès les années 1834-1838. La comparaison des manuscrits et du texte imprimé montre enfin comment, pour certains chants, le jeune éditeur a effectivement remplacé des mots par d'autres, et changé des noms propres pour donner une apparence historique et médiévale à des textes souvent beaucoup moins anciens ${ }^{10}$; il relie ainsi Le Faucon à une insurrection du début du $\mathrm{XI}^{\mathrm{e}}$ siècle. Dans l'ensemble, il est ainsi confirmé que le Barzaz-Breiz ne constitue pas une source, mais les carnets manuscrits peuvent en être une, de grande qualité et remontant parfois jusqu'au $\mathrm{XV}^{\mathrm{e}}$ ou au XIV ${ }^{\mathrm{e}}$ siècle.

Ce n'est pas tout à fait tout. En quelques années, et sans l'initiation d'aucun maître, ce n'est pas moins de cinq activités scientifiques différentes qu'a inaugurées le jeune La Villemarqué. Outre ce travail de collecte de textes de plusieurs années, il s'est préoccupé d'en fournir les mélodies, dont on sait l'importance pour soutenir la mémorisation. Il a fait un travail d'éditeur qui a eu le mérite de susciter en Bretagne des vocations à la collecte. Il s'est fait historien, puisqu'il a tenté de dater ses textes en les rattachant à des événements anciens. Il s'est fait philologue, enfin, en ce qu'il a initié la comparaison avec la poésie écrite galloise ${ }^{11}$. Excusez du peu.

Dans sa préface de 1839, il présente sa méthode d'élaboration des textes en soulignant la nécessité de recueillir le plus grand nombre possible de versions. Comme tel morceau s'avère tronqué ou altéré par le chanteur, l'éditeur peut se permettre une licence et " une seule ", celle " de substituer à certaines expressions vicieuses, à certaines strophes moins poétiques de cette version [...] les mots correspondant des autres leçons ». Il a suivi des cours à l'École des chartes, et peut-être n'a-t-on pas assez remarqué qu'il

7. LAURENT, Donatien, Aux sources du Barzaz-Breiz. op. cit., p. 291-292.

8. Des contradicteurs ont affirmé qu'au temps de sa jeunesse, La Villemarqué ne savait pas même le breton (ibidem, p. 23, 28, 38).

9. SAussure, Ferdinand de, Cours de linguistique générale, Paris, Payot, 1971, p. 45.

10. Laurent, Donatien, Aux sources du Barzaz-Breiz, op. cit., p. 286.

11. Hersart de La Villemarqué, Théodore, Barzaz-Breiz, op. cit., t. 2, p. 192. 
concevait l' " établissement ${ }^{12}$ " des textes oraux sur le modèle de l'édition des textes écrits : les versions des chanteurs sont altérées comme le sont les copies d'un manuscrit original et les copies de copies, et il s'agit de reconstituer l'original en effectuant des corrections. Pionnier, il n'avait pas pris pleinement conscience des spécificités de l'oralité et des méthodes critiques qu'elle requérait, lesquelles ont été élaborées par la génération suivante de collecteurs (pas d'embellissements, édition des variantes, indication systématique du lieu, de la date, de la collecte et du nom du chanteur). Finalement, c'est surtout le jeune historien qui n'a pas eu la prudence nécessaire et qui a poussé l'éditeur à des interpolations. Dans l'enthousiasme de ses vingt-quatre ans - son âge au moment de la première édition - et à partir de rapprochements rapides et ténus avec des sources écrites, il s'est permis, en effet ${ }^{13}$, de modifier ses textes de collecte pour conforter l'historicité et l'ancienneté qu'il leur attribuait.

\section{Le texte de collecte de Potred Plouiaou}

Venons-en au texte que nous éditons. À Plouyé, le dimanche de la pentecôte, un conflit éclate entre les paysans et les maîtres du sol; se sentant spoliés, des milliers de paysans en colère se rendent à Quimper, à 45 km au sud-ouest, où les maîtres de la ville les accueillent avec de la poudre à canon, si bien que les paysans prennent la ville et en brûlent les maisons.

La Villemarqué a recueilli ce chant en 1841 ou 1842 (dates de l'essentiel du deuxième carnet), de la bouche " d'un mendiant nommé Iouenn Vraz ${ }^{14}$ " (le grand Yves), à Plouyé ou aux environs, en Haute-Cornouaille, c'est-à-dire sur les lieux même de l'action.

Le texte manuscrit a été écrit sur une feuille volante pliée dont les deux moitiés, dans le carnet tel qu'il est relié aujourd'hui, sont séparées par des feuilles intermédiaires. Long de 120 octosyllabes ou vers courts, il est un peu plus long que le texte imprimé. Qu'il ait été noté en présence d'un chanteur est révélé par divers indices qui se situent aux deux étapes successives de son élaboration. D'une façon générale, l'écriture de l'ensemble est homogène et très rapide; les apostrophes de $c$ 'h, en particulier, sont soit de simples points au début du h, soit même sont élidées. À plusieurs reprises, le chanteur a hésité dans sa restitution du texte, chantant trop tôt un ou deux vers (vers $23 s q$ ), puis retrouvant les bons couplets que le collecteur a alors notés en marge (vers 19-22, d'une écriture descendante, faute de place) ou en interligne (vers 56-57). Le texte présente des éléments

12. C'est dans ce sens qu'il utilise l'expression d' " établir un texte ", que Luzel entend en faisant un contre-sens (LAURENT, Donatien, Aux sources du Barzaz-Breiz, op. cit., p. 24 note 7).

13. Luzel, François-Marie, De l'authenticité des chants du Barzaz-Breiz de M. de la Villemarqué, Saint-Brieuc, Paris, Brest, 1872. GouRviL, François, Théodore-Claude-Henri Hersart de La Villemarqué (1815-1895) et le Barzaz-Breiz (1839-1845-1867), Rennes, Oberthur, 1960.

14. Hersart de La Villemarqué, Théodore, Barzaz-Breiz, op. cit., t. 2, p. 20. 
de métrique ancienne comme la présence de tercets parmi les distiques. Les trois premiers vers, qui lancent une farouche malédiction au soleil, à la lune, à la rosée et à la terre, constituent une formule d'incipit qui se retrouve dans une autre complainte, trégoroise cette fois, recueillie par Luzel, celle d'Ervoanik al Lintier ${ }^{15}$. Cette formule figure également en tête de la version d'Al Lesvamm fall (la marâtre) que chantent les sœurs Goadec, de Treffrin, près de Mael-Carhaix ${ }^{16}$.

Puis La Villemarqué, intervenant en tant qu'éditeur, a modifié certaines formes; or parmi celles-ci, certaines sont caractéristiques d'un terroir. Les finales de pluriels en -aou, qui sont effectivement cornouaillaises, ont été corrigées en -ou. La finale du verbe kwezi (tomber) a été remplacée par la finale plus commune -a (kweza, vers 46). Tabluz (vers 43), forme inhabituelle pour tabut, ignorée des différents dictionnaires, est remplacée par strafil. Comme ailleurs dans le Barzaz-Breiz, des formes correctes ont été curieusement remplacées par d'autres qui le sont moins (emzivad > zivab, vinourez > minourez). Il est très significatif qu'un cri de défi vulgaire (Ni a ra fout, vers 91 , "on se fout... ») ait été noté, puis remplacé par une forme plus policée (Ni a ra fors); La Villemarqué tenait à montrer la tradition populaire comme exempte de toute trivialité, et le fait qu'il ait d'abord écrit une forme triviale suggère que celle-ci provient bien de son chanteur.

Le texte imprimé dans le Barzaz-Breiz donne un aperçu assez fidèle du texte manuscrit. Quelques additions de l'éditeur cependant, tout en étant ponctuelles, ne sont pas neutres car elles tendent à imputer l'iniquité des maîtres du sol aux Français habitant dans les villes. Au vers 54 du Barzazbreiz, "le sang des Français d'abord " est une interpolation à la place du " sang des seigneurs ". Au vers 60, "les os des Bretons " remplace " les reliques bénies ${ }^{17}$ ". Ces modifications résultent du projet de construction d'un sentiment d'appartenance nationale et bretonne qui était celui de La Villemarqué, et confirment que le texte initial n'est pas de lui. Un nom propre du Barzaz-Breiz, enfin, créait une confusion chronologique : l'évêque de Quimper qui tempère la rage destructrice des révoltés est nommé Rosmadec par le texte édité. Or le collecteur a d'abord noté une forme qui semble être Eskob madek ('évêque Madec, vers 93), puis il a écrit, en traits épais, Ros par-dessus Eskob, ce qui fait Rosmadec. Comme Bertrand de Rosmadec fut évêque de Quimper de 1416 à 1443, ce " coup de pouce " était une tentative de faire correspondre le chant avec une des dates ${ }^{18}$ données pour la prise de Quimper par Moreau, la seule source écrite alors connue.

15. LuZEL, François-Marie, Gwerziou Breiz-Izel : chants populaires de la Basse-Bretagne, Lorient, t. 1, 1868, p. 530, 542. GuILlOREL, Eva, 2010, p. 71 et 494.

16. C'est la gwerz bien connue de La femme aux deux maris (Ar plac'h hi daou bried) mais les deux versions qu'en donne Luzel ignorent cette formule.

17. Eskern ar Vretoned remplace ar c'hreireou benniget.

18. Moreau hésite sur le millésime : “ 1489, ou, selon d'autres, 1430 " (MorEAU, Jean, Histoire de ce qui s'est passé en Bretagne..., op. cit., p. 16). Toutes les dates indiquées sont incohérentes : aucun des deux jours précisés (mercredi 30 juillet pour la prise de la ville, dimanche 4 août pour la bataille) ne convient à aucun des trois millésimes, 1430, 1489 et 


\section{Un chant de 1490}

Il s'agit maintenant de situer ce chant historiquement. La ville de Quimper a été prise par des paysans de Cornouaille révoltés au début de l'été 1490 : Moreau hésite sur la datation, mais le fait et le millésime sont attestés par un registre de la chancellerie ducale, ainsi que par les comptes de dépense de la ville ${ }^{19}$. La date donnée par Moreau pour la prise de la ville, le 30 juillet, n'est pas en contradiction avec ce document. C'est la seule occurrence connue d'une prise de cette ville au cours d'une révolte, et cet événement conduit donc à dater le chant de l'été 1490.

En outre, un lien entre la prise de Quimper et la paroisse de Plouyé est confirmé par le chanoine Moreau. Celui-ci a rédigé son historique de la révolte en résumant un " ancien manuscrit " dont il disposait. À Plouyé vivait, entre 1481 et 1496 , un certain Louis Moreau ${ }^{20}$ qui pourrait bien avoir été un parent du chanoine et l'auteur de cet ancien manuscrit. Receveur de la seigneurie de Plouyé pour le vicomte de Rohan, et lui-même seigneur foncier, Louis Moreau fut non seulement un témoin oculaire de la révolte, mais aussi très probablement un des adversaires des paysans révoltés. Or cette " commune effrénée et en très grand nombre ", qui culmina avec la prise de Quimper, " prit sa source au terroir de Carahès ou Carhaix et du côté d'Huelgoat sous la conduite de trois frères paysans qu'on dit originaires de la paroisse de Plouyé21 ". Ces trois localités se trouvent dans le Poher, autrement dit la Haute-Cornouaille, un pays enclavé entre les Monts d'Arrée, au nord, et les Montagnes Noires, au sud. Ce ne sont évidemment pas seulement les habitants de Plouyé qui ont pris et pillé Quimper, mais cette paroisse est un des épicentres de la révolte, dans le résumé de Moreau comme dans le chant.

Quant au motif de la révolte, le chanoine Moreau indique seulement que les paysans revendiquaient " la propriété de leurs terres ". Aucun des trois détenteurs de fiefs qui résidaient à Plouyé n'y fut tué. Le chant rapporte un conflit entre les paysans et les seigneurs, maîtres du sol. Le dimanche de la Pentecôte arrive un " archer " qui ordonne que l'on prise les biens des

1490. On ne connaît pas d'évêque de Quimper nommé Madec. L'anthroponyme est par ailleurs bien connu et toujours porté.

19. Mandement de justice accordé le 18 août 1490 aux habitants de Quimper en raison de pilleries commises "par les gens de commune " de Cornouaille (Arch. dép. LoireAtlantique, B 12, fo $162 \mathrm{r}^{\circ}$, publié par LA BORDERIE, Arthur de, "Choix de documents inédits sur le règne de la duchesse Anne ", Bulletin et Mémoires de la Société archéologique du département d'Ille-et-Vilaine, t. 6, 1868, p. 243-349, aux p. 310-311).

20. Arch. dép. Morbihan 20 J 251, publié par NASSIET, Michel, "Émeutes et révoltes en Bretagne... ", art. cit., 1990, p. 155. Louis Moreau tenait un fief à Plouyé dès 1481 et était seigneur de Kerbiquet.

21. Moreau, Jean, Histoire de ce qui s'est passé en Bretagne..., op. cit., p. 16. Une rémission ducale a été accordée le 23 novembre 1490 à Jehan Lancien pour avoir insurgé « la comune contre la duchesse" (Arch. Dép. Loire-Atlantique, B 13, fo $64 \mathrm{v}^{\circ}$ ). L'ancien est une traduction de Coz. Or un tenancier nommé Yvon Coz est cité par le premier aveu de la seigneurie de Plouyé en 1517 (Arch. dép. Loire-Atlantique, B 1712). 
chefs de famille pour que ceux-ci déguerpissent. Cette expropriation est vécue comme une expatriation qui va empêcher de prier sur les tombes des parents. Son impiété est dénoncée par les ossements dans le cimetière qui se mettent à trépigner " comme s'ils étaient vivants " et ensevelissent l'archer. La colère éclate et les paysans décident d'aller à Quimper pour se "renseigner sur ce qui les attend ". Le chant associe donc étroitement une volonté farouche de lutte de classes et un sentiment religieux incluant la conception fantastique de la mort qui est bien connue dans la culture bretonne ${ }^{22}$.

Les habitants sont désignés soit par le mot tiek, qui signifie ménager, chef de ménage, soit par labourer, du français laboureur, paysan disposant d'une terre à cultiver. Ils sont victimes des seigneurs (otrounez) et des nouveaux nobles (tudjentil neo) ou des nobles des villes (dudjentil-ker). Le premier de ces "nobles nouveaux " pourrait bien être Louis Moreau, le receveur et fermier de la seigneurie. L'archer pourrait être un sergent apportant la sentence d'un tribunal car, en cette fin du $\mathrm{XV}^{\mathrm{e}}$ siècle, des procès opposaient des paysans à leur seigneur à propos du statut des terres $^{23}$. Comme l'a vu La Villemarqué, l'ordre de procéder au prisage fait penser que cette expulsion est perpétrée dans le cadre du domaine congéable, un régime agraire dans lequel « seul le seigneur foncier est propriétaire du fonds, tandis que le domanier est propriétaire des édifices et superfices $^{24}$ " (les levées des récoltes et les arbres des talus). Le domanier est fermier du sol et peut en être congédié par le seigneur foncier, ce qui est pour lui une cause de ruine. Un congément est à la fois une expulsion et une expropriation.

L'archer ordonne que tous les frais du prisage en incombent aux domaniers (" et que les frais soient à votre charge ${ }^{25}$ "). Les règles juridiques du domaine congéable étaient codifiées en des usements locaux, et Plouyé, au nord-ouest de Carhaix, se situe dans le pays de Poher sous l'" usement " duquel en effet le domanier devait acquitter tous les frais du prisage et payer non seulement son propre expert mais aussi celui du congédiant ${ }^{26}$. Les " usements " existaient dès le $\mathrm{XV}^{\mathrm{e}}$ siècle ${ }^{27}$ mais n'ont été mis par écrit que vers la fin du XVI ${ }^{\mathrm{e}}$ ou au XVII ${ }^{\mathrm{e}}$ siècle. Dans le chant, les ménagers estiment

22. Le BRAZ, Anatole, La légende de la mort chez les Bretons armoricains, $4^{\mathrm{e}}$ édit., Paris, 1922. CroIX, Alain, La Bretagne aux $16^{e}$ et $17^{e}$ siècles, la vie, la mort, la foi, Paris, Maloine, 1981.

23. NASSIET, Michel, "Émeutes et révoltes en Bretagne... ", art. cit., 1990, p. 148. GALLET, Jean, La seigneurie bretonne, 1450-1680, l'exemple du Vannetais, Paris, 1983, p. 447.

24. SEe, Henri, Les classes rurales en Bretagne du Xve e siècle à la Révolution, Brionne, Monfort, 1978, p. 269, 272, 274.

25. "Ar mijou diwar ho koust-hu " (vers 34).

26. GIRARD, Traité des usements ruraux de Basse-Bretagne, Quimper, 1774, p. 23-24; BAUDOUIN DE MAISONBLANCHE, Institutions convenancières ou traité raisonné des domaines congéables en général et spécialement à l'usement de Tréguier et de Goëlo, Saint-Brieuc, 1776, t. 2 p. $260 s q$.

27. Le vicomte de Rohan résume l'usement de sa vicomté en 1479 (TAILLANDIER, dom Ch., Histoire ecclésiastique et civile de Bretagne, Paris, 1761, p. CLXVII (art. LXVIII) et p. CCIX). 
être congédiés et chassés par leurs seigneurs en violation de la coutume : serait-ce alors que les seigneurs fonciers du Poher auraient introduit ce report de tous les frais sur les domaniers?

En outre, une spécificité de Plouyé était que les trois quarts environ de la paroisse étaient sous la seigneurie du vicomte de Rohan, alors que tout le pays alentour faisait partie du domaine ducal. Il est probable que le vicomte ait voulu détourner les impôts ducaux payés par ses sujets de la seigneurie de Plouyé comme dans ses autres seigneuries, comme nous allons maintenant le voir.

\section{Des chants de révolte}

Comparons ce texte avec une autre collecte de La Villemarqué qui a déjà fait l'objet d'une édition critique, le Faucon ${ }^{28}$. Le manuscrit se trouve dans le premier cahier de La Villemarqué; il n'en reste que la partie centrale, un ensemble de 38 vers. Le collecteur l'a recueilli auprès d'un vieux sabotier, nommé Brangolo, qui lui a chanté deux autres textes et qui avait pris part aux combats des Chouans. Devant les feux de la Saint-Jean, la rébellion commence par le refus de payer un impôt direct, sans doute exceptionnel. Le mot d'ordre est de porter un "feu rouge " sur "les valets du fisc ${ }^{29}$ ". Les acteurs sont des paysans qui sont notamment des éleveurs ${ }^{30}$. Ils descendent de la montagne et marchent sur " la ville de Rohan ", leur nombre passant de trois mille à trente mille. C'est ouvertement un chant de lutte et de défi ("Si c'est querelle et bataille qu'ils cherchent, Ils en trouveront avant que jaillisse le jour! ${ }^{31}$ »). Le texte donne la parole à un chef ou du moins une forte tête, "le grand Kado ", ainsi qu'à sa femme à ses côtés.

Le nom du lieu de destination, "la ville de Rohan ", "ker a Rohan " (vers 14), est précisé au cours d'une seconde récitation : e rohan > é stank rohan (vers 23). Stang-Rohan est en effet le nom d'une motte en Pluguffan, à six kilomètres à l'ouest de Quimper, laquelle était le chef-lieu du grand fief de Quéménet ${ }^{32}$ que les Rohan tenaient depuis Quimper jusqu'à la baie d'Audierne. Or un mandement ducal du 27 novembre 1490 accordé à un marchand de Quimper précise que seize paroisses " se insurgerent et s'assemblerent a grant numbre... en faits d'armes » et entrèrent dans Quimper dont

28. LAURENT, Donatien, "Autour du Barzaz-Breiz : Ar Falc'hon - Le Faucon, texte inventé ou chant recueilli? ", Bulletin de la Société archéologique du Finistère, t. 105, 1977, p. 333349. LAURENT, Aux sources du Barzaz-Breiz. op. cit., p. 182 et p. 297.

29. Tan ru war gwizien ar gwiriou. Ce vers comporte une rime interne et constitue donc un parfait octosyllabe de moyen Breton. Gwizien est le pluriel métaphonique de gwas (valet). Or dans son ouvrage, La Villemarqué a remplacé ce mot par potred (les gars), qui n'est guère approprié, s'agissant de valets du fisc, et qui fait disparaître la rime interne : il change dans le sens d'une banalisation.

30. "Mes troupeaux sont maigres", me chatal treut.

31. Ma de trous ha kan a glaskont, Ken ne strinko 'nn de a gaffont (vers 9-10).

32. TREvedy J., "Les fourches patibulaires du fief de Quéménet ", Bulletin de la Société archéologique du Finistère, 1883, p. 211-224, à la p. 217. 
ils pillèrent des maisons ${ }^{33}$. On ne sait si c'est le pillage attesté par l'acte du 18 août ou un second. Il s'agit d'un autre épisode de révolte, impulsé par les habitants d'un petit pays précis que le mandement situe en nommant trois des seize paroisses, Saint-Nic, Plomodiern et Plonévez-Porzay qui sont au bord de la baie de Douarnenez. Or c'est justement dans la paroisse voisine de cette dernière vers l'est, Quéménéven, que La Villemarqué a collecté ce chant. Ces paroisses appartenaient au Porzay, un autre fief des Rohan au nord du Quéménet. Ce mandement et Le Faucon situent donc tous deux leur action dans cet espace seigneurial des Rohan.

Or, pendant les guerres entre le duché de Bretagne et le roi, le vicomte de Rohan a pris parti pour le second et fut même le chef de l'armée royale en Bretagne en 1489. Dans ce contexte de guerre civile, entre 1487 et 1491, Rohan a détourné l'impôt public là où il le pouvait pour financer « les opérations militaires du parti francophile ${ }^{34}$ ". Il a fait lever notamment des fouages, c'est-à-dire un impôt direct qui pourrait bien être la taxe que refusent de payer les paysans du Faucon et qui les fait marcher sur StangRohan. Il semble donc que le Faucon date de ce contexte et des années 1488-1490; il est plausible même, sans qu'on en ait une preuve absolue, qu'il date de la révolte de l'été 1490.

La Bretagne avait connu au $\mathrm{XV}^{\mathrm{e}}$ siècle une prospérité que les guerres avec le roi mettaient en péril. Ces révoltés ne se pensent pas comme misérables, mais craignent de le devenir (" ma fortune est en train de se perdre, je vais être complètement ruiné ${ }^{35}$... »). Ce trait confirme l'hypothèse de Jean Meyer selon laquelle ce monde paysan se révolte " parce qu'il est sûr de lui-même, assez riche, à la fois, pour former une proie désirable mais aussi pour être, profondément, un corps social refusant toute atteinte à sa prospérité, si relative fut-elle ${ }^{36}$ ".

Il nous faut passer à une vision de synthèse sur la révolte de 1490. Une grande révolte paysanne était une succession de plusieurs épisodes, intervenus en plusieurs lieux et pour divers motifs. Les sources écrites montrent qu'il y eut au moins deux épisodes distincts : une sédition des paysans du Poher, pour un motif lié à la propriété du sol, commencée à Plouyé, et une marche des paysans du Porzay. Le texte des Gars de Plouyé se rapporte au premier, celui du Faucon pourrait se rapporter au second.

C'est un phénomène général, le premier de nos chants le confirme, que les villes ne s'associent pas aux révoltes paysannes et même se retournent contre elles. La ville de Quimper a été prise par une armée de paysans qui

33. Arch. dép. Loire-Atlantique, B 13, f 70 voo, publié par LA BorderIE, Arthur de, 1868, p. 311, et par Delumeau, Jean, Documents de l'histoire de la Bretagne, Toulouse, Privat, 1971, p. 148-149.

34. Kerherve, Jean, L'État breton aux XIV et XV siècles, Paris, Maloine, 1987, p. 591-593, d'après Arch. dép. Loire-Atlantique, B 51, fo 14.

35. "Mont a ra ma zanvez da goll, Da goll a ian enn holl d'an holl " (vers 3-4).

36. MEyER, Jean, DupuY, Roger, "Bonnets rouges et blancs bonnets ", Annales de Bretagne et des pays de l'Ouest, 82, 1975, t. 4, p. 405-426, à la p. 420. 
ont dû venir de divers pays; cette prise a pu intervenir à la date indiquée par Moreau, le 30 juillet. Les paysans révoltés ont accepté une bataille rangée en rase campagne le 4 août au cours de laquelle ils furent battus et massacrés par les nobles du pays et des soldats anglais au service de la duchesse ${ }^{37}$. Le 7 septembre cependant, des paysans révoltés s'assemblaient encore à Châteauneuf-du-Fou ${ }^{38}$. Cette bataille et cette défaite des paysans ont donné lieu à d'autres modalités de mémoire collective. La première est la toponymie : le pré dans lequel les paysans furent massacrés fut nommé le "pré de mille ventres ", et ce nom restait connu un siècle plus $\operatorname{tard}^{39}$. La seconde est un proverbe qui serait venu de cette bataille ("Tiens bon, fais ferme, Jean, et tu seras duc en Bretagne $\left.{ }^{40} "\right)$.

La datation de ces deux chants permet de les comparer avec le Chant du Rosemont qu'ils précèdent de seulement trente-cinq ans. Ce chant en français a été recueilli dans la première moitié du XIX ${ }^{\mathrm{e}}$ siècle et se localise au sud des Vosges. Les premiers éditeurs y ont vu un épisode de la guerre de Trente Ans, en raison d'autres traditions orales recueillies en même temps, mais l'identification du chef, Jean André, que l'on sait condamné à la décapitation en 1527, a permis d'y voir un épisode de la révolte des paysans du printemps $1525^{41}$.

La comparaison révèle une frappante analogie quant à la forme et au fond. Ces chants font allusion à une action collective de façon imagée, en particulier à une marche dirigée vers un but précis et nommé. Ils chiffrent et exagèrent le nombre des hommes assemblés pour montrer l'importance du mouvement. Le Faucon et Rosemont nomment un chef et en rapportent des paroles, si bien que leur auteur semble se situer parmi les révoltés euxmêmes - le Rosemont passant même plusieurs fois à la première personne du pluriel. Ils évoquent les motifs des révoltés avec compréhension.

Le Chant du Rosemont et Les gars de Plouyé ont aussi en commun de chacun se terminer par une expression allusive. Celle du Rosemont est même énigmatique : "S'ils avaient passé par Angeot/en revenant par Larivière/ tous les enfants du Rosemont/seraient devenus des sires. "Plouyé : "Retournons donc chez nous! En route!/Mais ç'a été pour leur malheur : ils ne sont pas tous arrivés chez eux. " Ces deux derniers vers semblent faire allusion à la bataille rangée qui fut une défaite. Elle incline à penser

37. Moreau, Jean, Histoire de ce qui s'est passé en Bretagne..., op. cit., p. 20.

38. D'après les comptes des miseurs de Quimper, Arch. dép. Finistère, E $1516, \mathrm{f}^{\circ} 9 \mathrm{v}^{\mathrm{o}}$. Moreau, Jean, Histoire de ce qui s'est passé en Bretagne..., op. cit., appendice $\mathrm{n}^{\circ}$ 1, p. 396. COUfFON, R., et WAQUET, H., "Extrait des comptes des miseurs de Quimper de 1478 à 1492 ", Bulletin de la Société archéologique du Finistère, t. 56, 1929, p. 83-89.

39. Prat mil goff (Moreau, Jean, Histoire de ce qui s'est passé en Bretagne..., op. cit., p. 20).

40. Dalc'h mat, Jan, sac'h, c'hui duc e Breis (ibidem, p. 21), notation inexacte qu'il faut lire Dalc'h mat, Jan, dac'h, c'hui vo duc e Breis.

41. Bischoff, Georges, "Contestations paysannes entre Vosges et Forêt Noire : la génération du Bundschuh (1493-1525) ", dans BRUNEL, G., BRUNET, S. (éd.), Les luttes anti-seigneuriales dans l'Europe médiévale et moderne, Toulouse, Presses universitaires du Mirail, 2009, p. 61-78. 
que la fin du Chant du Rosemont est aussi une allusion au tragique destin des révoltés qui furent taillés en pièces par la noblesse. Cette ressemblance formelle entre les deux textes de La Villemarqué et un chant des Vosges, que le collecteur breton ne pouvait pas connaître, est une preuve supplémentaire de l'authenticité des trois.

Enfin, la façon imagée de rapporter l'action collective, l'amplification du mouvement, la compréhension des motifs des révoltés, l'impression que donnent les chants de provenir du milieu même des révoltés pourraient être considérées comme des caractéristiques des chants de révolte.

\section{Dans la longue durée, mémoire ou auto-censure}

La permanence d'une propension à la rébellion dans un même espace et dans la longue durée a frappé les historiens, et c'est une problématique devenue classique que de tenter de l'expliquer. C'est notamment le cas de la Haute-Cornouaille qui connaît des séditions récurrentes sur plus de cinq siècles. En 1675, les habitants de vingt paroisses attaquèrent le château du Kergoët, en Saint-Hernin, un peu au sud de Carhaix, et les paysans révoltés tinrent le pays pendant plus de trois mois. Des émeutes eurent lieu à Spézet, paroisse où culminent les Montagnes Noires, en 1764, 1789, 1792, et c'est là aussi qu'en 1824 ou 1825, un propriétaire qui voulait y exercer son droit de congément suscita une grande émeute que La Villemarqué raconte en détails $^{42}$. Aux XIX ${ }^{\mathrm{e}}-\mathrm{XX}$ e siècles, les majorités électorales y furent le plus souvent de gauche ${ }^{43}$. En 2013, Carhaix fut l'épicentre de la contestation de la taxe carbone. Dans ce pays, un comportement de révolte ou de contestation s'est donc perpétué pendant cinq siècles. Comment l'expliquer?

Les auteurs du XIX ${ }^{\mathrm{e}}$ siècle décrivaient les tempéraments des habitants en fonction de leur environnement géographique et voyaient ceux des " montagnes " comme belliqueux ${ }^{44}$. L'histoire sociale du $x^{e}$ siècle a cherché des causes à ces comportements de longue durée dans les faits socio-économiques et les rapports de production. Il s'agit, en l'occurrence, du domaine congéable, dont on a beaucoup discuté s'il était un facteur de séditions et de contestations ${ }^{45}$. Tant Les gars de Plouyé que l'émeute de 1824 suggèrent que les congéments massifs révoltaient en effet les paysans.

42. Hersart de La Villemarqué, Théodore, Barzaz-Breiz, op. cit., t. 2, p. 287-289. Sur cette émeute, $c f$ une note de Le Bastard de Mesmeur dans son édition de MOREAU, Jean, Histoire de ce qui s'est passé en Bretagne..., op. cit., p. 18. La date et le nom du propriétaire, d'ailleurs notaire, sont donnés par LE MEN, "La révolte du papier timbré en Cornouaille ", Bulletin de la Société archéologique du Finistère, 1878, t. 5, p. 183-202, à la p. 200.

43. Dubreull, Léon, Les vicissitudes du domaine congéable en Basse Bretagne, Rennes, 1925. Michel, Jean-Yves, Religion et politique en Bretagne de 1850 à 1960. Le cas du Poher, Gourin, Keltia Graphic Editions, 2000, p. 221, 195.

44. Tandis que les habitants des vallées ont une "nature peu belliqueuse [...] dans les montagnes [...] le caractère est tout différent " (HERSART DE LA VILLEMARQuÉ, Théodore, Barzaz-Breiz, op. cit., "Avant propos ", p. Xv).

45. MEYER, DUPUY : " il est frappant de voir combien peu l'on parle du domaine congéable » ("Bonnets rouges et blancs bonnets", art. cit., p. 421 et p. 408). Sur la ville, p. 421. 
On peut légitimement se demander si la mémoire collective, ravivée par ces chants, n'a pas aussi contribué à cette reproduction des comportements de contestation. C'est ce que suggèrent les explications données par un vieillard "des montagnes " et rapportées longuement par La Villemarqué ${ }^{46}$ dans un texte peu connu parce qu'il n'est pas reproduit dans les dernières éditions :

" Je vais vous dire pourquoi il y a des chansons qu'on n'osait pas trop vous chanter; c'est que plusieurs d'entre elles ont une vertu, voyez-vous; le sang bout, la main tremble, et les fusils frémissent d'eux-mêmes, rien qu'à les entendre; plusieurs contiennent des mots et des noms qui ont la propriété de mettre l'écume de la rage à la bouche des ennemis des chrétiens, et de faire éclater leurs veines; quand nous les chantions en marchant contre les Bleus, nous voyions qu'ils les faisaient fringuer, comme de jeunes chevaux qui ont bu du vin de feu mêlé à de la poudre à canon; quand nous les dansions la nuit autour du feu du bivac [sic], dans quelque cour de manoir incendié par les républicains, nous entendions, vous ne croiriez pas? nous entendions nos fusils, nos bâtons et nos fourches de fer, rangés en faisceaux derrière nous, s'agiter d'eux-mêmes et murmurer comme s'ils eussent été impatientés de rester au repos..."

Cette animation des fusils et des fourches, ainsi que la conscience de son caractère fantastique ("vous ne croiriez pas "), sont analogues à celle des ossements dans Les gars de Plouyé (vers 76-77). En marchant contre les soldats de la République, les Chouans ne chantaient donc pas seulement des chants nouvellement composés mais aussi de vieux chants héroïques, ce type de chants dont l'existence est discutée par la critique. Et selon ce vieillard, qui en parle d'expérience, certains de ces chants exhortaient irrépressiblement à la révolte (il faut entendre leur "vertu " dans le sens ancien de force agissante). On devine ici deux processus mémoriaux analogues à ceux que Youenn Le Prat peut observer de façon beaucoup plus détaillée dans le corpus des chants bretons de la Contre-Révolution ${ }^{47}$.

De vieux chants porteurs d'une note héroïque étaient l'objet d'une réactivation lors d'une lutte ultérieure; leur charge symbolique leur permettait de souder la troupe des révoltés et de servir de signe de ralliement. Déjà dans Le Faucon ${ }^{48}$, la femme du grand Kado " chantait en marchant ${ }^{49}$ ".

46. Hersart de La Villemarqué, Théodore, Barzaz-Breiz, op. cit., t. I, "Avant propos ", p. XVI. Ce vieillard ne serait-il pas Brangolo, le chanteur du Faucon?

47. LE PRAT, Youenn, "Chanter contre. Usages politiques et mémoriels de la chanson vernaculaire contre-révolutionnaire en Bretagne ", communication au colloque de Caen de 2013.

48. "Hag hi a gané tréma ié " (vers 29).

49. Trois siècles plus tard, en 1793 à Saint-Pol-de-Léon, lors du tirage au sort en vue de trouver des recrues, et selon Lan Inisan (1826-1891), c'est en chantant les strophes du Faucon qu'un groupe de jeunes gens marcha vers la place occupée par les soldats républicains. "Nous formâmes tous un cortège en chantant ensemble et en nous répondant d'un groupe à l'autre, tous les deux couplets... " La précision de ce fait permet-elle d'y voir un souvenir authentique? " Nous entendîmes un groupe arriver sur la grand-place, en chantant cette complainte, composée au temps jadis, et qui se trouve dans un livre intitulé Barzaz-Breiz » (INISAN, Lan, La bataille de Kerguidu et autres événements survenus 
À l'inverse, les explications du montagnard montrent que le répertoire était l'objet, de la part des chanteurs, d'un processus de sélection qui pouvait compromettre la transmission des textes considérés par trop dangereux. Cela expliquerait qu'il n'en ait pas été collecté d'autres. Ce témoignage permet de revenir sur un problème méthodologique dont l'enjeu est de première importance, celui des chants qui n'ont été collectés que par un seul chercheur. Pour Luzel, c'est le premier motif d'y voir un faux ${ }^{50}$. Cet argument était extrêmement fort pour les auteurs critiques du dernier tiers du XIXe siècle. Or on connaît aujourd'hui plusieurs chants effectivement collectés qui n'ont été trouvés que par un seul chercheur : Le Faucon, les Gars de Plouyé, et d'autres chants collectés par La Villemarqué ${ }^{51}$ et par d'autres collecteurs ${ }^{52}$.

Cette question de l'unicité de certaines collectes est centrale car elle touche à l'essence même de la méthode d'enquête de terrain. Les informateurs, en effet, ne chantent pas à tout le monde. Il est significatif que La Villemarqué, lui aussi, se soit heurté au mutisme de gens des campagnes tout en ayant l'intuition qu'ils en savaient davantage : le montagnard se taisait, " et le plus souvent il souriait de cet air narquois et important qu'il prend volontiers quand il veut montrer qu'il n'est pas dupe ${ }^{53}$ ". D'une part, le jeune collecteur était inconnu et " le montagnard est défiant "; il a fallu l'aide du " presbytère " et du " manoir " pour faire tomber la méfiance. D'autre part, comme nous l'avons vu, certains chants qui dénonçaient tel ou tel étaient considérés comme dangereux, et pour ceux qu'ils dénonçaient, et pour ceux qui les chantaient, et étaient l'objet d'une rétention.

L'expérience de la collecte de terrain confirme ces difficultés ${ }^{54}$. Elle montre que pour recueillir certains textes plus rares et plus secrets que

en Basse-Bretagne pendant la Révolution en 1793, Paris, R. Laffont, 1977, p. 160). Lan Inisan dit raconter la vie d'un personnage qui serait son grand-père paternel.

50. LuZEL, François-Marie, " Documents inédits sur le mouvement populaire connu sous le nom de "la révolte du papier timbré», en Basse-Bretagne ", Bulletin de la Société archéologique du Finistère, t. 14, 1887, p. 35-67; LuZEL, François-Marie, " Réponse de M. Luzel ", ibid., p. 152-162.

51. C'est le cas de deux autres textes du premier carnet : Merlin-Barde et Les Chouans (LAUREnt, Donatien, Aux sources du Barzaz-Breiz. op. cit., p. 287-302). Ce pourrait être le cas enfin de la pièce la plus contestée de l'édition de 1839, La Prédiction de Guiclan; elle manque dans les manuscrits, mais l'informatrice du collecteur, la chanteuse Clémence Penquec'h, s'en souvenait encore " très bien " en 1906, comme l'a constaté la nièce de La Villemarqué (Laurent, Donatien, Aux sources du Barzaz-Breiz. op. cit., p. 285 et p. 326). Au surplus, il existait dans les campagnes des éléments de traditions orales sur un prophète nommé Guinglan (NASSIET, Michel, "La littérature bretonne et l'histoire ", art. cit., p. 51, note 47).

52. La complainte sur l'assassinat de la dame de Kerizel en 1663 n'a pas été trouvée par Luzel parce qu'elle ne s'est pas diffusée en dehors de la commune d'Yvias (GIRAUDON, Daniel, "Itron a Gerizel ", Bulletin de la Société d'Emulation des Côtes-du-Nord, 1984, p. 60-77).

53. Hersart de La Villemarqué, Théodore, Barzaz-Breiz, op. cit., t. I, " Avant propos ", p. XV.

54. LAURENT, Donatien, "La Villemarqué et les premiers collecteurs en Bretagne ", in La Bretagne et la littérature orale en Europe, Mellac, Brest, CRBC, CRDLO, CIRCTO, 1999, p. 153-167, aux p. 163-164. 
d'autres, il faut "y croire ", rester attentif et ouvert à l'inattendu, montrer que l'on connaît bien ce dont on parle, créer éventuellement les conditions d'un défi. Comptent également la façon de se présenter et d'entrer en matière, les introductions que l'on fait valoir, la réputation qui vous a précédé, la manière d'interroger... Toutes ces variables jouent, et c'est pourquoi deux chercheurs explorant un même terrain de recherche d'une même tradition n'obtiendront pas les mêmes résultats.

Pour conclure, les textes manuscrits des Gars de Plouyé et du Faucon sont très sûrs mais le second est très incomplet. Ce sont tous deux des chants de révolte : ils épousent le point de vue des révoltés et sont ardemment porteurs de revendication sociale. Le premier date précisément de la prise de Quimper intervenue à l'été 1490; le second peut dater de la même révolte, mais peut se situer aussi dans les années 1487-1491. Ils gardent la mémoire des motifs des paysans révoltés ainsi que de leurs actions. Les analogies de ces deux textes avec le Chant du Rosemont de 1525 sont particulièrement significatives et confirment qu'ils sont relativement contemporains.

Bien que restreint, ce corpus montre que le genre des chants de révolte et, plus largement, des chants politiques, a existé en langue bretonne. Ces chants ont transmis des textes et des éléments de mémoire collective sur près de quatre siècles. La tradition orale bretonne avait une grande liberté et, à l'occasion, pouvait prendre un caractère ouvertement subversif. Dès lors, il est probable qu'il a été composé d'autres chants de ce genre mais qu'ils n'ont pas été recueillis, notamment parce qu'ils faisaient l'objet de réticences de la part des chanteurs, qu'ils réveillaient des motifs de conflits et rappelaient les divisions de la communauté. 


\section{Potred Plouiaou}

[Archives de La Villemarqué. Deuxième carnet]

\section{Documents numérisés par le CRBC (UBO-BREST).}

Nos plus grands remerciements vont à la famille Hersart de La Villemarqué pour la confiance qu'elle nous a toujours accordée et la générosité avec laquelle elle nous a prêté ses manuscrits.

Texte établi et traduit par Donatien Laurent

Principes d'édition : La transcription en langue bretonne est la reproduction du manuscrit. Le signe "/" signifie que par-dessus une première leçon, ici écrite à gauche de ce signe, a été notée par le collecteur une seconde, ici écrite à droite. En outre sont proposées, en italique ou en note, quelques restitutions de vers qui sont suggérées par un mot ajouté par le collecteur au cours d'une seconde audition et/ ou par la nécessité de la rime. La traduction inclut ces restitutions.

[au crayon, de l'écriture de Pierre de La Villemarqué : p. 174]

\section{Naik Lukas, Lesvam ${ }^{55}$}

1 Malloz d'ann heol, ha d'al loar,

$$
\text { ft malloz }
$$

2 Malloz ar/Ha d'ar c'hlouiz a gwez war ann [douar;

3 Malloz d'ann douar, ha d'ar parkou,

$4 \mathrm{~A}$ zo penn abek d'ar strivaou/gwall [strifou;

kiriek

$5 \mathrm{~A}$ zo penn abek

a zo d'ar strivaou/fou kiriek

6 Etre ar/Tre ann otrou hag ann tiek.

7 A lak ar strabull war ar mez;

8 A lak mer a hini diaez

9 Meur 'emzivad, hag intanvez,

10 meur a vinour a vinourez
1 Maudits soient le soleil et la lune,

2 Maudite soit la rosée qui tombe sur la terre,

3 Maudits soient la terre et les champs

4 Qui sont la cause des disputes/terribles disputes

5 Qui des disputes sont responsables

6 Entre le seigneur et le maisnager,

7 Qui mettent le trouble dans les campagnes, 8 Qui en mettent plus d'un dans la gêne,

9 Qui font plus d'un orphelin et d'une veuve, 10 Mettent plus d'un fils et d'une fille en tutelle,

55. Titres de complaintes recueillies par La Villemarqué et qui présentent les mêmes vers de malédiction que ci-dessous. Ceux-ci servent d'introduction aussi à « Ervoanik Al Lintier ", collectée par LuzeL, François-Marie, Gwerziou, 1868, p. 542. 


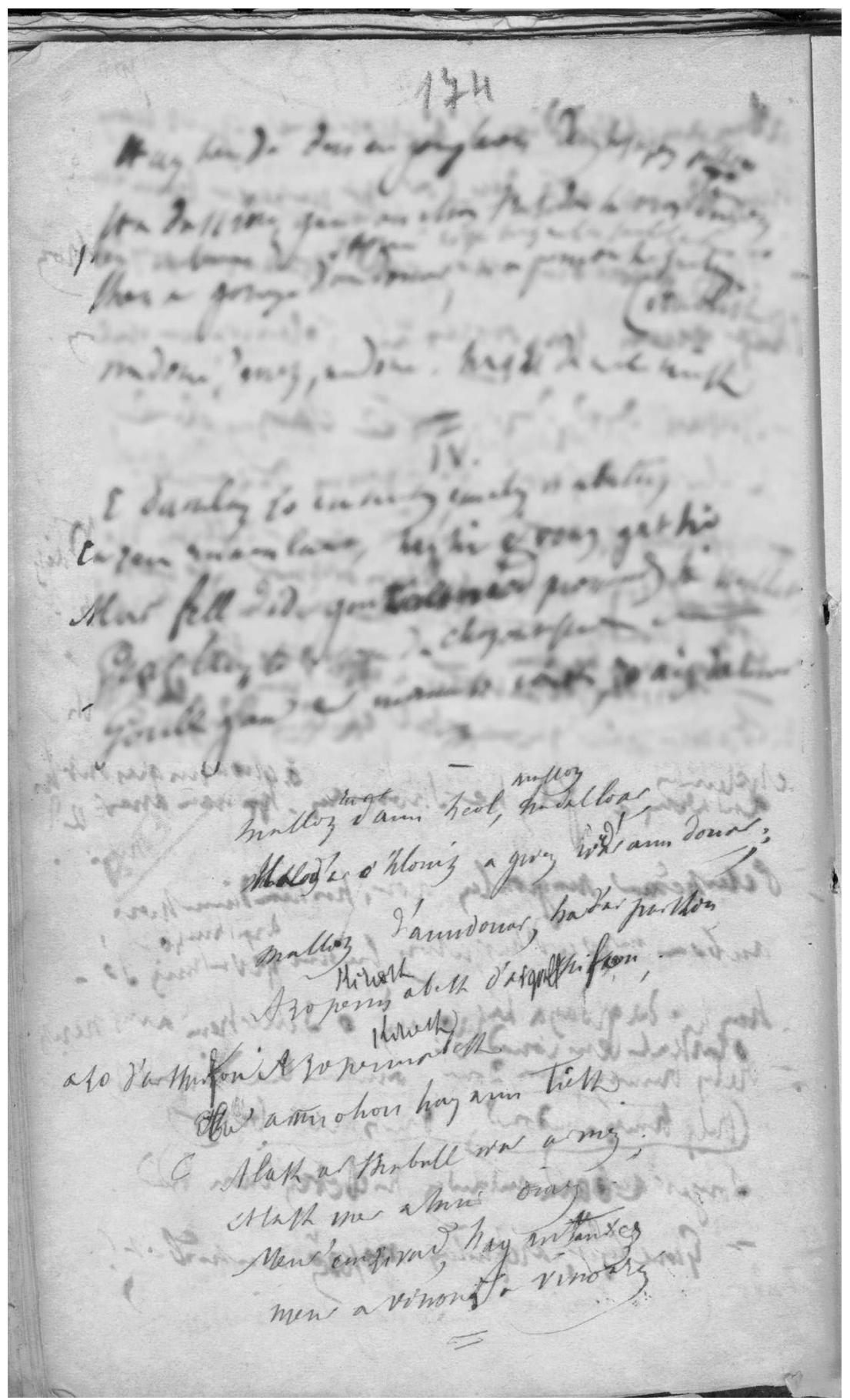


[au crayon p. 175]

11 meur a gredur war ann henchou,

12 o c'houlen/Gand he vamm o skuilla [daelou.

13 Mill Malloz d'ann dud/chentil ker, 14 A ra bec'h war al labourer

15 Dutchentil ker, tud gentil/nemet tudo [Gall

tugentil

16 o kas/klask digas giziou/kichou bro [c'hall

les deux vers suivants entre parenthèses :

17 o kas distruja/diskarga kichou breiz

18 /o/Lakas/t digas/traou neve/wez flamm [direiz.

$$
\text { trarall/traou all }
$$

En face, en marge, les quatre vers suivants :

19 Disul gwen, goude 'nn ofern bret.

20 Digou/wet ann arzer er veret;

21 war diri ar groaz ann arzer

22 he zalgat o tevi ter

les deux vers suivants entre parenthèses :

23 Mede, war ar groaz ann arser

24 war groaz ar veret ann arzer

$26 \mathrm{Ma}$ aru/de ann arser penn ma si,

25 he l/zaoulagad ru o tevi; ter

27 he zaoulagad ru o tevi

28 vel d'eur poudad dour o tevi/firvi,

29 - chilaouet holl potred plouiaou/ieaou,

30 Chilaouet ar c'hourc'hemennaou;

31 Evid ar bloaz hag eun dé bloc'h

32 Ra brizor pez a berc'hen d'hoch.

$33 \mathrm{Ra} /$ Prizor ho tier hag ho stu, 34 Hag ar/Ar mijou diwar ho koust hu.

$35 \mathrm{Ma} \mathrm{v/zeot} \mathrm{pa} \mathrm{gerfet} \mathrm{gand} \mathrm{ho} \mathrm{tud,}$

$36 \mathrm{Ha} / \mathrm{Gand}$ ho arc'hant gen-hoc'h da glask

[ho klud/t.

37 Argand er ialc'h da glask ho klud.
11 Plus d'un enfant sur les routes

12 Avec sa mère qui verse des larmes.

13 Mille fois maudits soient les gentilshommes de ville,

14 Qui oppriment le laboureur.

15 Gentilshommes de ville, mais des gens de France,

16 Qui cherchent à apporter les coutumes de France,

17 Qui font détruire les coutumes de Bretagne, 18 Qui apportent des nouveautés choquantes

19 Le dimanche de Pentecôte, après la grand'messe,

20 Arrive l'archer dans le cimetière

21 Sur les degrés de la croix, l'archer,

22 Les yeux enflammés de colère,

23 Sur la croix était l'archer

24 Sur la croix du cimetière, l'archer

25 Les yeux brûlants de colère

26 L'archer est arrivé au bout de ma maison

27 Avec les yeux rouges qui brûlaient

28 Comme un pot d'eau bouillante

29 - Écoutez tous, gars de Plouyé,

30 Écoutez les commandements :

31 D'ici un an et un jour entier,

32 Qu'on prise ce qui vous appartient!

33 Qu'on prise vos maisons et vos fumiers ${ }^{\mathrm{a}}$ 34 Et que les frais soient à votre charge.

35 Et allez, quand vous voudrez, vous et les vôtres,

36 Avec votre argent, vous chercher un perchoir!

37 La bourse pleine, vous chercher un perchoir!

a. Nous gardons la traduction métaphorique du mot stu par La Villemarqué, mot qui désigne toutes les valeurs économiques mises en cause dans la culture des terres chaudes, c'est-à-dire fertiles, depuis les amendements et les fumiers que l'on met en terre au moment du labour, jusqu'à la valeur de la levée des céréales au printemps. 
Potred Plouiaou (1490) et la question des chants de révolte en langue bretonne

175

108

mew a-grodur war am honoton,

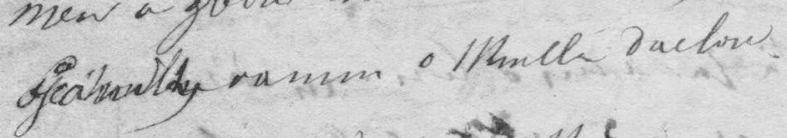

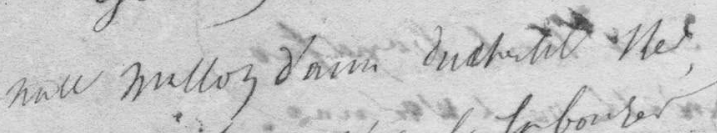

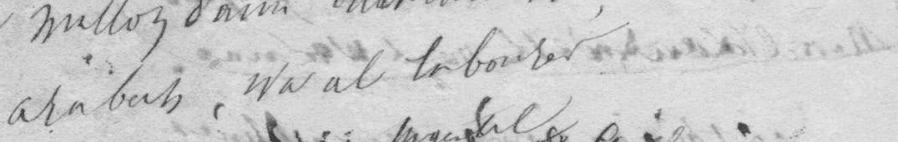

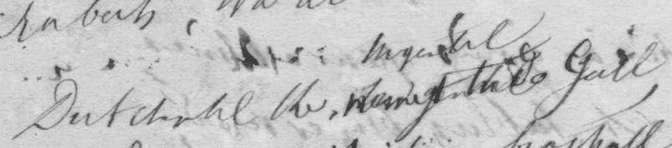

oldasidegas thighion fiounale.

- Prlm Distariza Dicton bierz

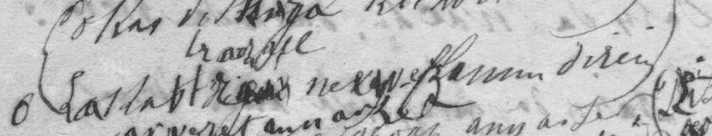

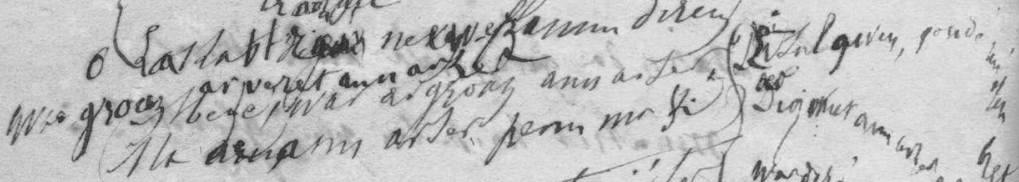

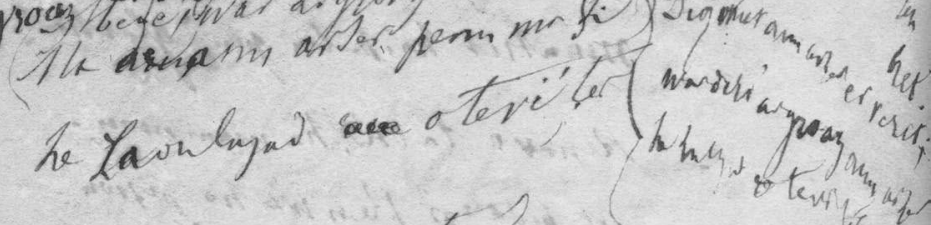
Le tainlyation

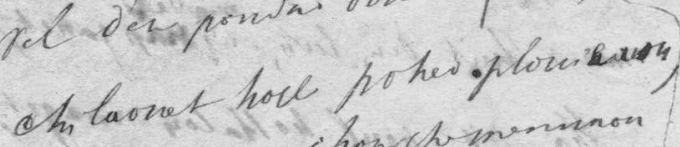
chiturnet ar i hountomeminon

$\Rightarrow$ critadfleng xny emmide floch in fingor tog a Eeration atwo ih.

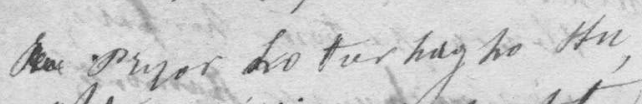

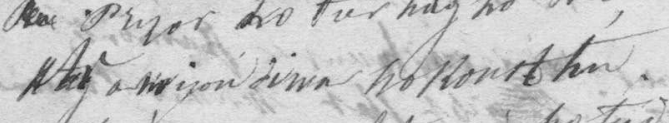

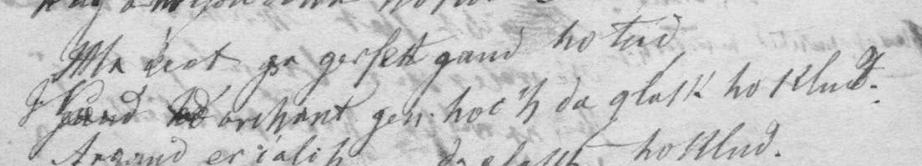

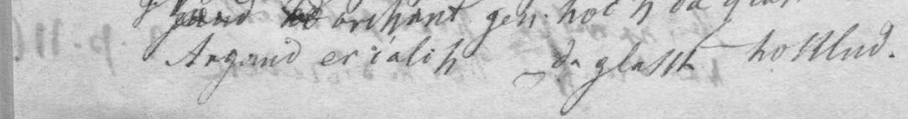

45 
[au crayon p. 176]

les trois premiers vers entourés :

$38 \mathrm{~Pa}$ zav ann deliou enn dero

39 Kent evit sevel e/baz an/er fao ${ }^{\mathrm{a}}$

40 War dek mervel a ra nao ${ }^{b}$

41 Mervel a eure dek war nao

42 oa ket ar gomz peur achuet, 43 Savet tabluz braz er vered;

44 Ann ezec'h,/tud koz ha iounk da groza;

45 Darn da wac'ha, darn da wela :

46 Darn da gwezi war ann douar,

47 Mantret ho c'halon gand glac'har:

48 - Kenavo, tadou, ha mammou;

$49 \mathrm{Na}$ bedomp ken war ho peziou.-

50 Ret eo deomp, monet divroet,

51 ni mont breman ${ }^{c}$

52 Mont kuit deuz lec'h, euz omp ganet; 53 omp bet ${ }^{\mathrm{d}}$

54 Ha war poul ho kalon maget;

55 Hag etre ho ta/tiou vrec'h douget.

Les deux vers ci-dessous insérés en interligne:

56 Kenavo sa/ent ha sentezet 57 Kenavo patrom benniget

- $($ Mec'hed Plouiaou ne welet ket $)=$

58 Kenavo patrom hor p/barrez

$59 \mathrm{Ni}$ zo war nent/enn hent ar j/paourentez

38 Quand les feuilles poussent sur le chêne 39 Avant de pousser sur le hêtre,

40 Sur dix hommes il en meurt neuf!

41 Il en mourait dix sur neuf.

$42 \AA$ A peine il achevait ces mots

43 Qu'une grande querelle éclata dans le cimetière.

44 Les hommes mariés, vieux et jeunes, se mirent à gronder;

45 Les uns criaient, les autres pleuraient;

46 Certains tombaient à terre,

47 Le cœur brisé par la douleur :

48 - Au revoir, pères et mères,

49 Nous ne pourrons plus prier sur vos tombes.

50 Il nous faut partir, exilés,

51 Partir maintenant (exilés)

52 Quitter les lieux où nous sommes nés

53 Quitter les lieux où nous sommes (élevés)

54 Et avons été nourris au creux de votre cour

55 Et portés dans vos bras.

56 Au revoir, saints et saintes,

57 Au revoir, patrons bénis,

58 Au revoir, patron de notre paroisse,

59 Nous sommes sur le chemin de la misère!

Les deux vers suivants en marge à gauche,

face aux vers :" Ni zo war nent...":

60 Potred Plouiau dez lavaret :

61 Tevet, merc'hed ne welet ket

60 Les gars de Plouyé ont dit :

61 - Taisez-vous, les filles, ne pleurez pas,

62 Ne welet ket merc'hed Plouiaou,

$63 \mathrm{Ne}$ welet ket, bugaligaou;

64 Ken na welfet gwad peb tiek,

$65 \mathrm{War}$ dreuj/sou dor he di o redek v. p. 116

62 Ne pleurez pas, filles de Plouyé,

$63 \mathrm{Ne}$ pleurez pas, petits enfants,

64 Avant de voir le sang de chaque maisnager

65 Couler sur le seuil de sa porte!

a. Ces deux parfaits octosyllabes formulent un dicton de Basse-Bretagne : " Ann deliou zigor en dero/ Kent evid digeri er' fô " : " les feuilles s'ouvrent sur le chêne avant de s'ouvrir sur le hêtre " (SAUvE, L.F., Proverbes et dictons de la Basse Bretagne, Champion, 1878, p. 110).

b. On retrouve ces vers non pas dans l'édition de Paotred Plouieo mais au début de "L'Hermine ", Barzaz Breiz, 1846, t. 1, p. 338, vers 1, 2 et 7.

c. Vers 51 probable : Ret eo deomp ni mont breman divroet.

d. Vers 53 probable : Mont kuit deuz lec'h omp bet ganet. 
Potred Plouiaou (1490) et la question des chants de révolte en langue bretonne

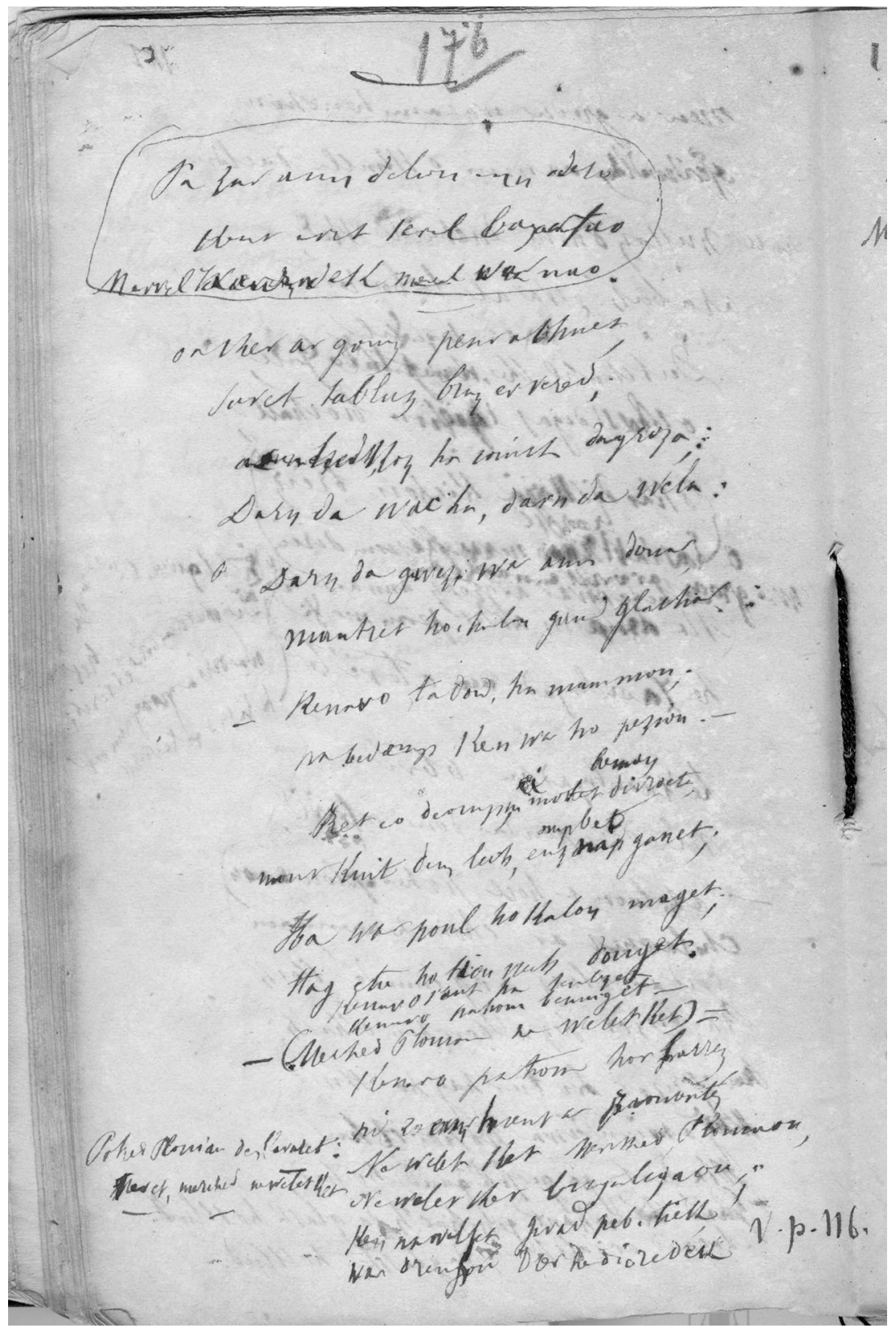


[au crayon p. 204]

v. p. 101 [au crayon: les jeunes hommes de Plouyé]

66 Welfet ar banné divézan

67 Gwad ann otrounez da ge/gentan -

68 Ann arzer pan n deves klewet/az

70 evel pa glevas

69 Meurbet ema bet souezet eslamet/ $\left[\mathrm{mmaz}^{\mathrm{a}}\right.$,

72 o/Wié daré pelec'h tec'het.

73 e/'vel den arog he fenn, n'euz gret,

74 Barz ar garnel, ema lammet.

75 Etouez ar c'hreireou/iou benniget.

76 Hogen klevet ar seurt burzud.

77 Ar c'hererou a/' zrask evel ann dud

78 Ar c'heriou a zo/zo em unanenet;

$$
\text { a zav, }
$$

79 A eneb d'an arzer war ho zreit;

80 ha chetu han peur d/ziflamet

81 ha chetu he galon rannet,

82 Hag hen, didan! hé/ho enteret/douaret.

83 Potred Plouiaou a lavaré.

84 - Deomp ni da c'hout hor zigaré.

85 E ger/traon Kemper pa erruzont, 86 ho otrounez a c'houlenjont.

87 - Porzer digoret ann noriou.

88 - Ma iamp da gomz euz hon aotrou.

89 = Eet al lezé koz tieien,

90 Ma na keret d/c'hweza poult gwenn Mar
66 Avant de voir la dernière goutte, 67 Mais le sang des seigneurs d'abord!

68 L'archer, en entendant ces mots, 69 En fut extrêmement surpris;

70 L'archer, lorsqu'il entendit,

71 sauta au bas de la croix

72 Il ne savait où fuir.

73 Il a fait comme quelqu'un qui a perdu la tête,

74 Il a sauté dans l'ossuaire

75 Parmi les ossements bénis.

76 Mais écoutez la sorte de prodige:

77 Les ossements trépignent comme s'ils étaient vivants!

78 Les ossements se sont unis,

79 Dressés sur leurs pieds, contre l'archer.

80 Et le voilà complètement démembré,

81 Et voilà son coeur brisé

82 Et lui enterré sous eux.

83 Les gars de Plouyé disaient :

84 -Allons apprendre sur ce qui nous attend!

85 En arrivant dans la basse-ville de Quimper, 86 Ils demandèrent à parler à leurs seigneurs.

87 - Portier, ouvrez les portes,

88 Que nous allions parler à notre seigneur.

89 - Allez-vous en, vils maisnagers,

90 Si vous ne voulez sentir l'odeur de la poudre blanche!

a. Ces additions suggèrent la restitution de deux vers : 70 Ann arzer evel pa glevas et 71 Diwar zez ar groaz a lammaz. 
Potred Plouiaou (1490) et la question des chants de révolte en langue bretonne

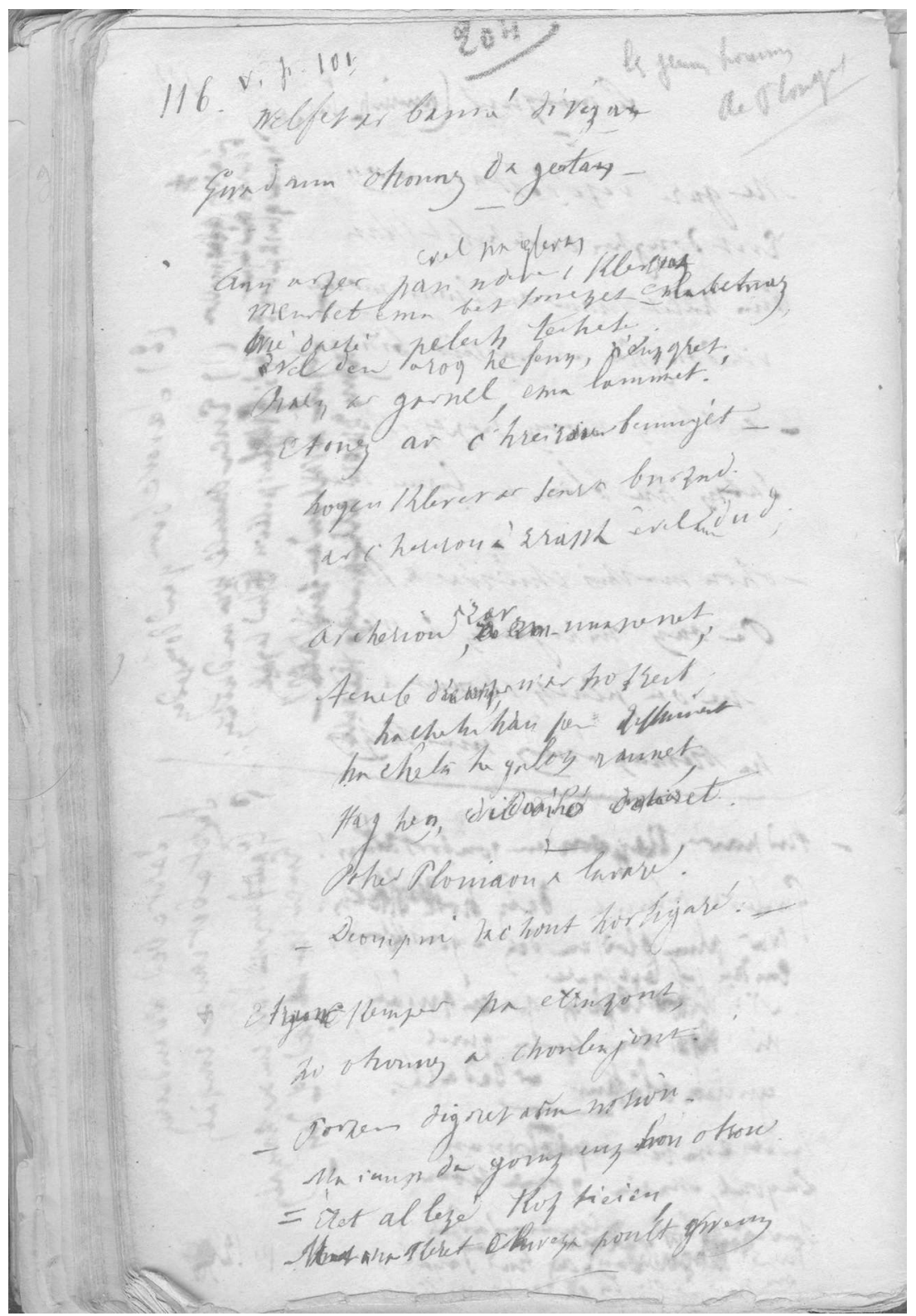


[au crayon p. 205]

91 ni a ra fout gant ho poult gwen 92 Kemet a ramp dez ho b/perc'hen. _

93 oa ket ho c'homz peur achuet,

94 An.../Tregont anezo zo lazet 95 Daou uget/Pevar ugent tanfoueltret

$96 \mathrm{Da} . . /$ Tregont (ann ezo) a zo lazet [annhé,

97 Daou/Pevar mil all a zo lammet tré

98 Daou mil a zo lamet er ger

99 Hag ann tan enn ker/en hi fol ha ter/e [kalz a sier

100 Ken a larent/grient : ah iaou! a iaou! 101 Terc'homp kuit rag potred Plouiaou,

102 ann tan (lakont) enn ti/barz ar/er ger [leiz a sier

103 Nemet enn hini/ti eskop Kemper

104 Ti Ann otrou escob/Ros madek kez

105 A zo/Zo mad d'ann dud paour diwar [mez

$106 \mathrm{~A} / \mathrm{a}$ zo denjentil a voad roial, Breiz ${ }^{\mathrm{a}}$ 107 à voad v/breton ha naren gall.

109 Hag a zac'h/zalc'h mad ér gizou reiz

110 ann eskob mad ho alié

111 Biou ann tier pa dremené :
91 Nous nous foutons de votre poudre blanche 92 Autant que de leur propriétaire!

93 Ils n'avaient pas fini de parler

94 Que trente d'entre eux sont tués 95 Quarante/quatre-vingt renversés

96 Que trente d'entre eux sont tués

97 Deux/Quatre mille autres avaient sauté à l'intérieur.

98 Deux mille ont sauté dans la ville 99 Et le feu en ville/fou et violent/dans beaucoup de maisons.

100 Tant qu'ils criaient : ah iaou! ah iaou! 101 Fuyons devant les gars de Plouyé!

102 Ils mirent le feu dans la maison/dans la ville pleine de maisons

103 Sauf dans la maison de l'évêque de Quimper;

104 Le cher monseigneur l'évêque Madec

105 Qui est bon pour les pauvres gens de la campagne

106 Qui est gentilhomme de sang royal 107 De sang breton et non pas français.

108 Qui est homme du sang des rois de Bretagne

109 Et qui tient bon aux coutumes justes.

100 Le bon évêque les conseillait

111 En passant à côté des maisons

\section{Graphie de Rosmadek (détail du bas de la page 205)}

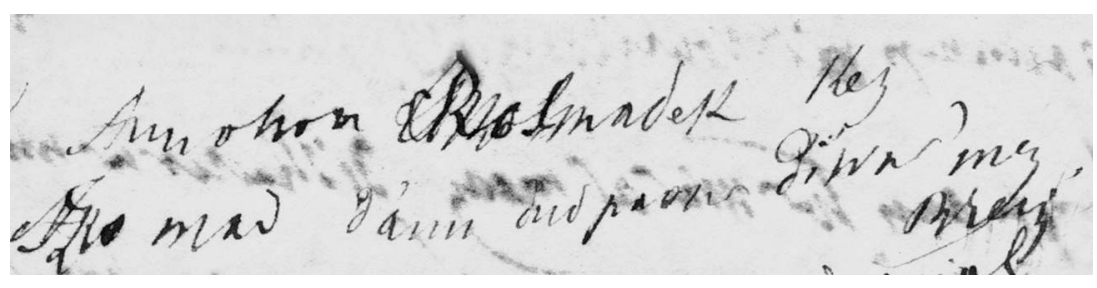




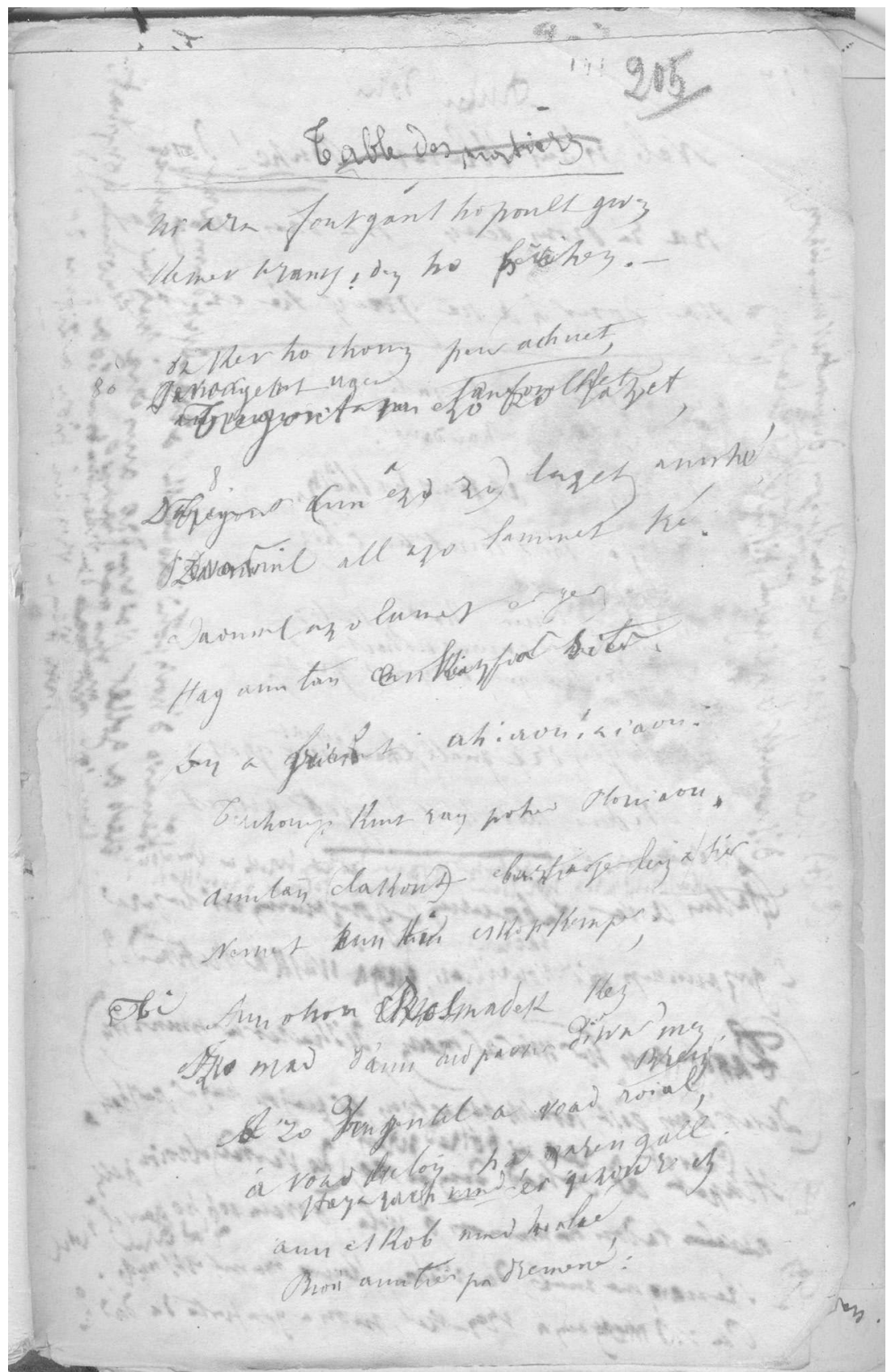


[au crayon p. 206]

112 arzet arzet, ma bugale, 113 arzet arzet enn han doué!

114 Ma bugalé, distroet enn dro/it war ho kiz, $115 \mathrm{Na}$ vezo ket torret ar c'hiz.

116 Potret Plouia ho dez sentet, 117 ha d'ar ger emant distroet

118 Distroi d'ar ger o dezont gret;

119 Hogen, dez/dre gwall, e.../chans dez [ont gret

eus int

$120 \mathrm{Ne}$ m/dint ket holl d'ar ger arruet,
112 Arrêtez, arrêtez, mes enfants, 113 Arrêtez, arrêtez, au nom de Dieu!

114 Mes enfants, retournez chez vous, 115 La coutume ne sera plus violée.

116 Les gars de Plouyé ont obéi 117 Et chez eux ils sont retournés

118 Ils sont retournés chez eux;

119 Mais c'est pour leur malheur qu'ils l'ont fait,

120 Ils ne sont pas tous arrivés chez eux... 
Potred Plouiaou (1490) et la question des chants de révolte en langue bretonne

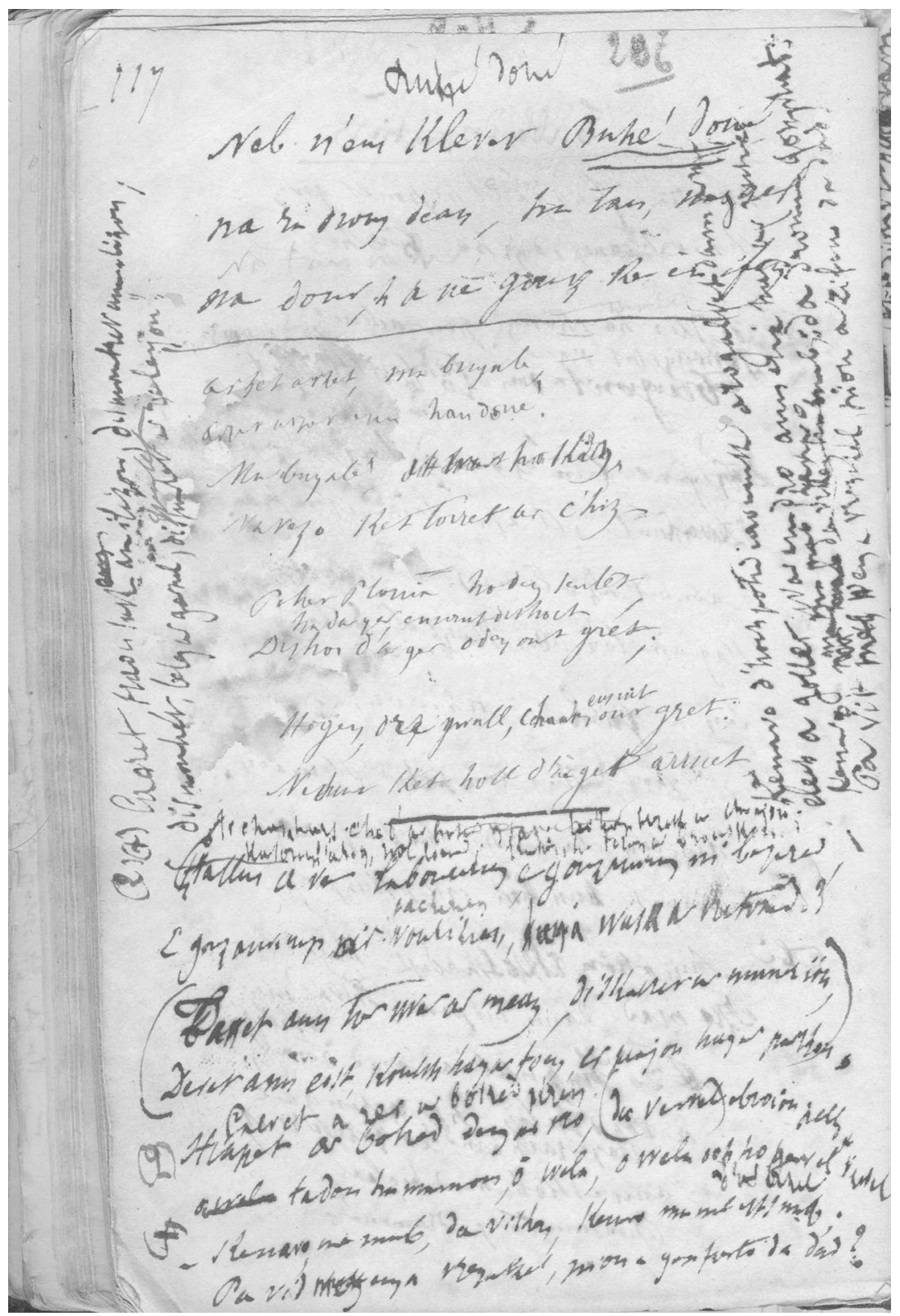




\section{RÉSUMÉ}

Cet article est l'édition critique du texte de Potred Plouaiou tel qu'il est transcrit dans le manuscrit d'Hersart de La Villemarqué qui l'a édité en 1845. La critique externe et interne montre que ce texte résulte bien d'une collecte de terrain auprès d'un chanteur. L'épisode qu'il rapporte, la prise de Quimper par des paysans révoltés, ne peut se situer qu'en 1490. L'article le compare ensuite avec deux autres chants de révolte, l'un en breton datant de la même révolte, l'autre, en français, de la Guerre des paysans de 1525; les ressemblances formelles entre ces trois textes apportent une preuve supplémentaire de leur authenticité. Ce dossier permet aussi de revenir sur un problème méthodologique, celui des chants qui n'ont été collectés que par un seul chercheur. Il montre que le genre des chants politiques a bien existé dans la littérature orale en langue bretonne.

\section{ABSTRACT}

This paper is a critical edition of the Breton text of Potred Plouaiou, taken from the manuscript of Hersart de La Villemarqué, who published it in 1845. External and internal criticism demonstrates that the text was the result of field research undertaken with a singer. The event it describes, the storming of Quimper city by rebel peasants, can only be dated to 1490. This paper compares the text with two other revolt songs, a Breton one, from the same context, and a French one, from the Peasants' War of 1525; the formal similarities provide additional evidence of the authenticity of all three. This case enables us to re-examine a methodological problem: the way to analyse songs collected by a single scholar. It underlines that the genre of political songs was part of the Breton oral literary tradition. 